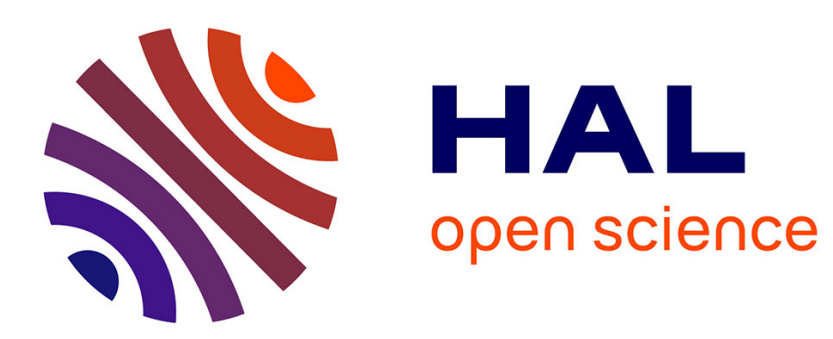

\title{
Apparent biaxiality due to oriented defects in samples of lamellar liquid crystals \\ Y. Galerne
}

\section{To cite this version:}

Y. Galerne. Apparent biaxiality due to oriented defects in samples of lamellar liquid crystals. Journal de Physique Lettres, 1979, 40 (4), pp.73-76. 10.1051/jphyslet:0197900400407300 . jpa-00231572

\section{HAL Id: jpa-00231572 https://hal.science/jpa-00231572}

Submitted on 1 Jan 1979

HAL is a multi-disciplinary open access archive for the deposit and dissemination of scientific research documents, whether they are published or not. The documents may come from teaching and research institutions in France or abroad, or from public or private research centers.
L'archive ouverte pluridisciplinaire HAL, est destinée au dépôt et à la diffusion de documents scientifiques de niveau recherche, publiés ou non, émanant des établissements d'enseignement et de recherche français ou étrangers, des laboratoires publics ou privés. 


\title{
Apparent biaxiality due to oriented defects in samples of lamellar liquid crystals
}

\author{
Y. Galerne \\ Laboratoire de Physique des Solides $\left({ }^{*}\right)$, Université de Paris-Sud, Bât. 510, 91405 Orsay, France
}

(Reçu le 26 juin 1978, révisé le 7 décembre, accepté le 21 décembre 1978)

\begin{abstract}
Résumé. - On étudie la biaxialité apparente d'un échantillon de cristal liquide lamellaire contenant des défauts orientés. La biaxialité est calculée dans le cas de dislocations coin parallèles les unes aux autres. On montre également que dans les phases lyotropes, la lumière diffusée reste trop faible pour masquer le phénomène. Pour finir, on tente une comparaison avec l'expérience [10].

Abstract. - We study the apparent biaxiality of a sample of a lamellar liquid crystal in homeotropic orientation and containing oriented defects. The biaxiality is calculated in the case of edge dislocations parallel to each other. We show also that in lyotropic liquid crystals, light scattering is very weak and does not obliterate the conoscopic image. We also make a comparison with the experimental results of ref. [10].
\end{abstract}

Most physical experiments such as those of optical indices give information which is integrated over a macroscopic volume. This unavoidable integration is not troublesome if the sample is homogeneously oriented. However if the sample is not homogeneously oriented, the measurement can be affected. In particular the symmetry of the measurement can be different from the symmetry of a well oriented sample. Here, we study the case of uniaxial phases of lamellar liquid crystals (such as, for example, smectic A and cholesteric for the thermotropic system [1], and $\mathrm{L}_{\alpha}$ phase for the lyotropic system [2]) which can lead to spurious biaxiality measurements. This happens if oriented defects like edge dislocations are created in the sample.

1. Edge dislocations. - In figure 1 is shown an edge dislocation of Burgers vector $\mathbf{b}$. Two edge dislocations of opposite sign attract each other but do not annihilate

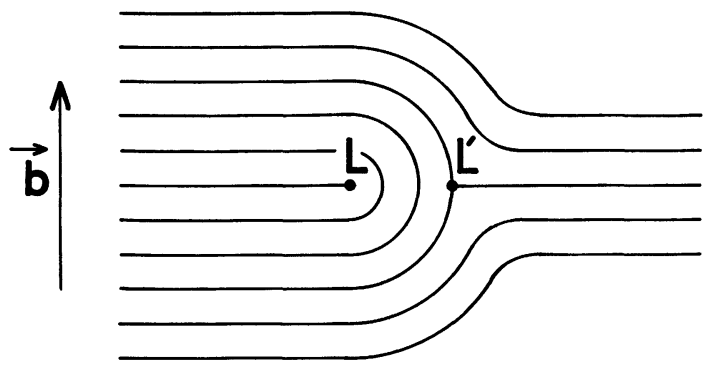

Fig. 1. - Edge dislocation of Burgers vector $b . L^{\prime}$ and $L^{\prime}$ are two disclination lines of opposite rotation $(+\pi)$ and $(-\pi)$. Only the layers are drawn.

(*) Laboratoire associé au C.N.R.S. (L.A. 2). and results in coupled edge dislocations as shown in figure 2. The existence of such a situation is clear in thermotropic liquid crystals [3] ; but in lyotropics it has to be restricted to Burgers vectors larger than $0.1 \mu$ which is the order of magnitude of the smallest observable edge dislocations [4], [5].

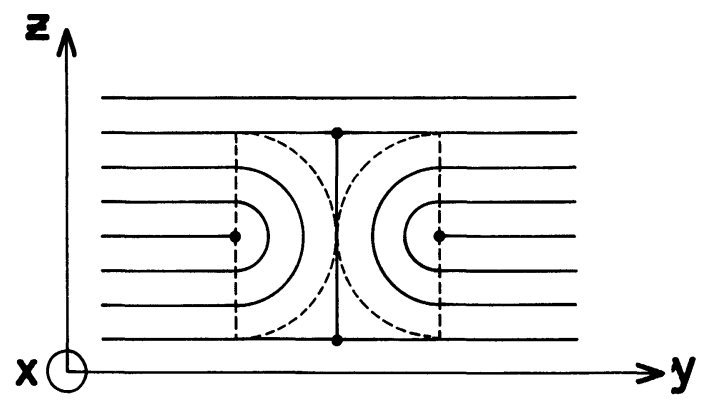

Fig. 2. - Coupled edge dislocations of Burgers vector of opposite sign. The exact situation is less schematic and the layers undulate to release the dilative stress. This pattern shows simply the origin of the structural biaxiality we discuss here.

A proof is the existence of oily streaks [1] which are commonly observed in homeotropic samples of the two types of phases, thermotropics and lyotropics. These oily streaks are interpreted [3] as undulations of the layers occurring when the dilative stresses around the coupled edge dislocations are larger than the undulation threshold [6]. When the dilative stresses remain below this threshold, no undulation is produced. If then, the Burgers vector of the coupled edge dislocations is smaller than the light wavelength, 
nothing is visible under an optical microscope and the sample looks perfectly homeotropic.

When the dilative stresses around the coupled edge dislocations are still stronger, far above the threshold, the undulations develop strongly and give rise to chains of focal conics [7]. Experimentally this case occurs very often in the thermotropics. In the lyotropics the situation seems more balanced. A reason for this, is the relatively lower compressibility modulus of the layers [8] which results in a dilative energy too weak to convert the coupled edge dislocations into focal conics. Another explanation is related to the fact that focal conics have to emit edge dislocations of small Burgers vector to build up [7]; and this seems especially difficult in lyotropic liquid crystals.

These edge dislocation in $\mathrm{L}_{\alpha}$ phases are observable and measurable by electron microscopy using different techniques such as freeze-etching [5], [9] or drying on filter paper [4]. The measured values of the Burgers vector range from $0.1 \mu$ to $1 \mu$.

When a shear is applied in the $x$-direction to homeotropic samples of $\mathrm{L}_{\alpha}$ phase, oily streaks are produced [10] parallel to this $x$-direction (the exact mechanism is not clear); the alignment of the samples is then homeotropic except near the core of the coupled edge dislocations. (Conversely and consistently with the ideas mentioned above, shear effects in thermotropics seem to lead directly to focal conics [11]. For this reason, our discussion has practically to be

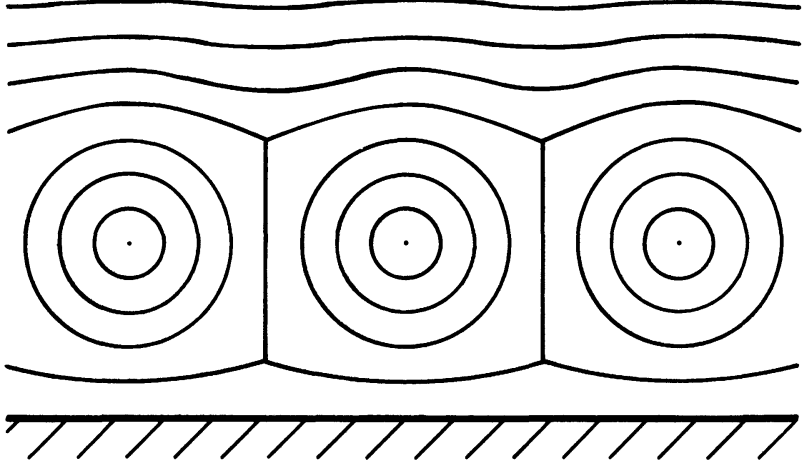

Fig. 3a. - Schematic representation of a coating of parallel coupled edge dislocations stuck at the glass surface.

They are represented with equal Burgers vectors for simplicity.

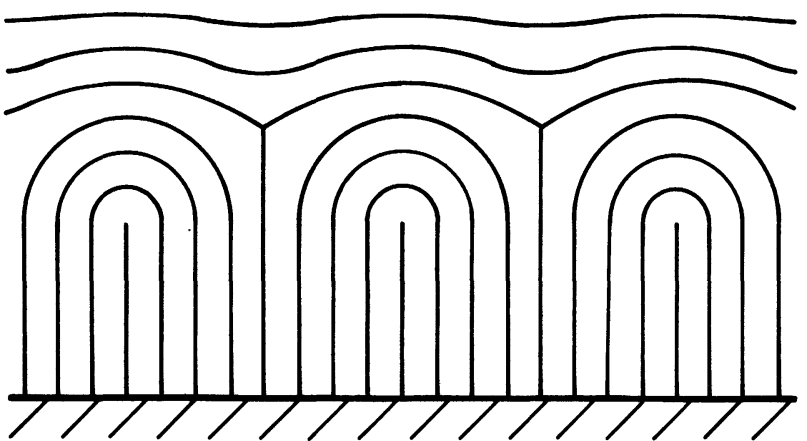

Fig. 3b. - Schematic representation of a coating of parallel coupled edge dislocations with planar anchoring conditions. reduced to the $\mathrm{L}_{\alpha}$ phases.) We can imagine several possibilities for the disposition of these edge dislocations. They may gather into sheets stuck at the glass surfaces, as is schematically shown in figure $3 a$ for an homeotropic anchoring, or as in figure $3 b$ for a planar anchoring. They may also be randomly dispersed in the bulk. In reality, it does not matter very much since the resulting effect, the apparent biaxiality is about the same for these three cases.

2. Calculation of the apparent biaxiality. - Let us consider a homeotropic sample of $\mathrm{L}_{\alpha}$ phase with one edge dislocation parallel to $x$ as in figure 2 . The Burgers vector b being in the range $0.1 \mu$ to $1 \mu$, this defect is not easily observable with the optical microscope. A very light thread will appear when focussing. Its brightness is proportional to $(\Delta \varepsilon)^{2}$ [12], i.e. to $(\Delta n)^{2}(\Delta \varepsilon$ being the difference between the extraordinary and the ordinary dielectric constants and $\Delta n$ being the birefringence). If we compare to the case of the thermotropics, when $\Delta n \simeq 0.1$ compared to $\Delta n \simeq 10^{-2}$ for the $\mathrm{L}_{\alpha}$ phases, this means that the edge dislocations of same Burgers vector will appear 100 times less bright in the $\mathrm{L}_{\alpha}$ phases than in the thermotropics.

So now let us estimate the conoscopic figure [13] given by this schematic sample. Each point $\mathbf{P}$ of the conoscopic screen corresponds to an incident plane wave. Each elementary volume increases the phase delay of the two polarizations $x$ and $y$ of this plane wave according to its own index ellipsoid. All the rays emerging from the different parts of the sample interfere in $\mathbf{P}$, separately for the two polarizations. The two electric fields resulting in $\mathbf{P}$ are proportional to $\left\langle\exp j \varphi_{i}\right\rangle$; $(i=x$ or $y$ depending of the considered polarization; the mean value is taken over all the transmitted rays and $\varphi_{i}$ is their respective phase delay). Provided that the variations of $\varphi_{i}$ are much smaller than $2 \pi$ (and this is the case if $b \Delta n \ll \lambda$ ), we can approximate :

$$
\left\langle\exp j \varphi_{i}\right\rangle \simeq \exp j\left\langle\varphi_{i}\right\rangle .
$$

This result shows that the transmitted rays give rise to a plane wave of wave-vector parallel to the incident wave-vector. There is no lens effects; and therefore, the light reaches $P$. This equation shows also that the resulting conoscopic figure is analogous to that produced by an ideal homogeneous sample with spatially constant index values. The transmitted plane wave is then polarized to produce the interference between the $x$ and $y$ polarization. This two-waves interference gives the light intensity for each point $\mathbf{P}$ on the screen.

The problem therefore reduces to the estimate of the ideal spatially averaged sample. This is easy to calculate for the simple case of figure 2 : let us assume that the sample is perfectly homeotropic except inside the dashed half circles of diameter $b$; and 
moreover that inside these half circles the orientation of the molecules (which is perpendicular to the layers in the $\mathrm{L}_{\alpha}$ phase) is radial. For the molecules making an angle $\theta$ with $z$, the dielectric tensor is :

$$
\left[\begin{array}{ccc}
\varepsilon_{0} & 0 & 0 \\
0 & \varepsilon_{0}+\Delta \varepsilon \sin ^{2} \theta & \Delta \varepsilon \sin \theta \cos \theta \\
0 & \Delta \varepsilon \sin \theta \cos \theta & \varepsilon_{0}+\Delta \varepsilon \cos ^{2} \theta
\end{array}\right]
$$

where $\varepsilon_{0}$ is the smaller principal dielectric value and $\varepsilon_{0}+\Delta \varepsilon$ the larger one. The mean value in the dashed volume is :

$$
\left[\begin{array}{ccc}
\varepsilon_{0} & 0 & 0 \\
0 & \varepsilon_{0}+\frac{\Delta \varepsilon}{2} & 0 \\
0 & 0 & \varepsilon_{0}+\frac{\Delta \varepsilon}{2}
\end{array}\right] .
$$

Averaging over the whole sample leads to :

$$
\left[\begin{array}{ccc}
\varepsilon_{0} & 0 & 0 \\
0 & \varepsilon_{0}+\frac{\alpha}{2} \Delta \varepsilon & 0 \\
0 & 0 & \varepsilon_{0}+\left(1-\frac{\alpha}{2}\right) \Delta \varepsilon
\end{array}\right] .
$$

where $\alpha$ is the ratio of the disturbed volume (dashed forms) over the sample volume.

The whole sample then appears to be biaxial, with a biaxiality :

$$
n_{y}-n_{x} \simeq \frac{\alpha}{4} \frac{\Delta \varepsilon}{n_{0}} \simeq \frac{\alpha \Delta n}{2}
$$

where $\Delta n=n_{z}-n_{x}$, is the birefringence. (The birefringence is not sensibly modified.) This biaxiality gives two optical axes in the $x z$ plane, i.e. parallel to the direction of the dislocation lines.

Now we suppose that the sample contains a lot of parallel edge dislocations as mentioned above. Provided that we neglect the interference perturbation produced by such a grating, we calculate, in the same way, the mean value for the two electric fields for each point of the conoscopic screen. We obtain the same dielectric tensor where $\alpha$ is the ratio of the whole disturbed volume (due to all the dislocations) over the sample volume. So the result stays the same. Nevertheless, a slight modification comes in when the dislocations join the glass surface with a planar orientation (case of Fig. 3b). The planar part must be taken into account. If we define by $\beta$ its relative volume, the measured biaxiality becomes then :

$$
n_{y}-n_{x}=\left(\frac{\alpha}{2}+\beta\right) \Delta n \text {. }
$$

In reality, the conoscopic image on the screen is the superposition of this interference effect and of the light scattering due to all the edge disclination. As mentioned above, this stray light is proportional to $\alpha(\Delta n)^{2}$ and therefore is of less importance than in thermotropics.

At this stage, we must emphasize that biaxiality does not prove the existence of edge dislocations inside a sample of $L_{\alpha}$ phase. Other kinds of oriented defects can produce biaxiality like, for example, elongated focal conics, undulations as described in ref. [5], or wrapped cylinders [14]. But a common feature holds. Since biaxiality arises from stable defects, it will persist for a long time, leading to a memory effect.

3. Experimental comparison. - In light of this rather conjectural model, a comparison with the experiment of S. Micciancio and F. Rondelez [10] is possible. And we propose the following interpretation : the shear applied on a homeotropic sample of $\mathrm{L}_{\alpha}$ lecithin produces oily streaks parallel to the shear direction $(x)$; this means that edge dislocations are produced parallel to $x$. Then curing the sample by heating at $95^{\circ} \mathrm{C}$ makes the most visible defects almost completely disappear as can be seen with a microscope; but probably, small invisible defects persist. Therefore, the sample still appears to be biaxial with the optical axes parallel to the shear direction. Numerically, the measured biaxiality is $n_{y}-n_{x}=10^{-4}$ which, for an usual birefringence value $\Delta n=10^{-2}$ in $\mathrm{L}_{\alpha}$ lecithin, corresponds to a small relative volume $\alpha=2 \times 10^{-2}$ for the disturbed region. If we interprete this value in the case of figure $3 a$, we get $\alpha=2 b / L$ where $b$ is the diameter of the cylinders and $L$ is the sample thickness. For a typical sample [10], $L=20 \mu$; then we get $b \simeq 0.2 \mu$. This corresponds to dislocations of about 40 layers. Note that the light scattered by such a disturbed region does not destroy the conoscopic pattern. Due to the $(\Delta n)^{2}$ effect, this stray light is the same as that produced by only one misaligned layer in a thermotropic liquid crystal sample.

In conclusion, we propose an interpretation for the experiment of shear induced biaxiality in $\mathrm{L}_{\alpha}$ lecithin, based on the existence of an invisible disturbed part of the sample, most probably localized near to the surfaces and oriented by the shear. Our model for the memory effect, the direction and the order of magnitude of the shear induced biaxiality is in good agreement with the experimental results and observations. This interpretation has the advantage of explaining the occurrence of biaxiality without any tilt of the conoscopic cross, as is realized by the experiment.

We would like to thank M. Kléman and J. Rault for very helpful discussions. 


\section{References}

[1] Friedel, G., Ann. Phys. 18 (1922) 273.

[2] Luzzati, V., Tardieu, A., Ann. Rev. Phys. Chem. 25 (1974) 79.

[3] Rault, J., C. R. Hebd. Séan. Acad. Sci. 280B (1975) 417.

[4] Kleman, M., CollieX, C., Veyssie, M., Advances in Chemistry series no 152, Ann. Chem. Soc. (1976) 71.

[5] Kleman, M., Williams, C., Costello, M. J. and GuliKKRZYwiCKI, T., Phil. Mag. 35 (1977) 33.

[6] Ribotta, R., Durand, G., J. Physique 38 (1977) 179.

[7]. Rault, J., Phil. Mag. 34 (1976) 753.

[8] Delaye, M., Ribotta, R., Durand, G., Phys. Lett. 44A (1973) 139.
[9] Williams, C., Thèse d'Etat (1976) Orsay.

[10] Micciancio, S., Rondelez, F., J. Physique Lett. 39 (1978) L-5. [11] Kleman, M., HoRN, R., Communication in Madonna (Italy), Ann. Phys. 3 (1978) 229.

[12] De Gennes, P. G., C. R. Hebd. Séan. Acad. Sci. 266B (1968) 15. [13] Born, M., Wolf, E., Principles of Optics (Pergamon Press, Oxford) 1964, 2nd ed.

[14] Papahadjopoulos, D., Vall, W. J., Jacobson, K. and Poste, G., Biochim. Biophys. 394 (1975) 483. 